\title{
SOFT X-RAY MAGNETIC SCATTERING STUDY \\ OF THIN FLIMS AND MULTILAYERS
}

BNL- -46497

\author{
C.-C. KAO, E.D. JOHNSON, J.B. HASTINGS, D.P. SIDDONS DE91 017472
}

National Synchrotron Light Source

Brookhaven National Laboratory, Upton New York 11973

C. VETTIER

Rerisot he

Institute Laue-Langevin, 38052 Grenoble CEDEX, France ALE 261991

\begin{abstract}
A brief discussion of the resonant magnetic scattering process is given. Results from recent studies of thin Fe films and $\overline{F e} / \mathrm{Gd}$ multilayers are used as examples to demonstrate the information can be obtained and the unique features of this technique: large resonant enhancement, sensitivity to magnitization, elemental specificity, and tunability of the penetration depth. Comparison is made with related techniques: magneto-optical Kerr effect, Faraday effect, and magnetic circular dichroism.
\end{abstract}

\section{Resonant magnetic scattaring}

Recently, large resonant enhancement of the magnetic scattering cross se tion near an absorption tinreshold has been observed in Ho and UAs [1,2]. In favorable cases the magnetic scattering cross section can be comparable to that of charge scattering. These observations can all be explained by the resonant exchange scattering process [3]. To summarize briefly, the large resonant enhancement is the result of strong electric multipole transitions from atomic core $1 ;$ is to unoccupied states above the Fermi level, while the sensitivity to magnetization arises both from spin polarization and from exchange splitting of the conduction band. In fact, in many respects resonant magnetic scattering can be considered as a simple extension of normal magneto-optical 
effects to higher photon energies. However, there is an important distinction berween the two cases. At lower energies, especially when both the initial and final states are in the conduction band, the spin-orbit splitting is often small in comparison with the bandwidth of the joint density of states, which leads to partial cancellation of the magneto-optical effects. On the other hand, at higher energies where the spin-orbit splitting of the initial core state is typicaily much greater than the bandwidth of the conduction band, the cancellation is small, which in turn makes the observed magneto-optical effects much more pronounced [4].

In the remainder of this paper, we will limit our discussion to dipole transitions and to the transverse scattering configuration, where the magnetization of the sample is perpendicular to the scattering plane. For a complete analysis of multipole transitions and polarization dependence, see reference [3]. Following Hannon et al. [3], the scatiering amplinile due to resonant exchange scattering in the transverse configuration, and considering only dipole transitions, is given by equation (1),

$$
f_{E l}^{(r e s)}=\frac{3 \lambda}{8 \pi}\left\{\left(\vec{e}_{f} \cdot \vec{e}_{o}\right)\left[F_{11}+F_{1-1}\right]-i\left(\vec{e}_{f} \times \vec{e}_{o}\right) \cdot \vec{z}_{j}\left[F_{11}-F_{1-1}\right]\right\}
$$

In equation (1), $\vec{e}_{o}$ and $\vec{e}_{f}$ are the polarization vertors of the incident and scattered beams, $\vec{z}_{j}$ defines the quantization axis, and $\mathrm{F}_{\mathrm{LM}}$ is the resonant response, where $\mathrm{L}$ is the order of the transition ( $\mathrm{L}=1$ for dipole transitions) and $M$ is the change in angular momentum $(\Delta M=0, \pm 1)$. The first term is the usual resonant scattering amplitude in anomalous scattering and the second term is the linear magnetic term, which is proportional to the magnetization of the sample. Since $\Delta \mathrm{M}$ equals $-1(+1)$ for left- ( right- ) circularly polarized light, the two terms in equation (1) can also be considered as the sum and difference of scattering due to left- and right-circularly polarized components of plane polarized light.

The relationships between resonant magnetic scattering and other related 
techniques can be briefly summarized with the aid of equation (1). Resonant magnetic scattering makes use of the coherent interference between the second term and first term in equation (1), and measures the total scattering intensity. On the other hand, the Faraday effect and magnetic circular dichroism measure the real part and the imaginary part of the second term, respectively. Moreover, since the real and imaginary parts of the scattering factor are related through Kramers-Kronig relations, the results from Faraday effect and dichroism measurements can be interconverted by performing Kramers-Kronig analysis. With both the real and imaginary parts of the scattering factor, the resonant scartering intensity can also be obtained [5]. This unique possibilty of cross referencing results from three different experimental techniques provides not only a much more stringent test for these results, but also an opportunity for them to complement each other.

\section{Case I: thin Fe film}

The specular reflectivity of p-polarized soft $x$-ray near the Fe $L_{I I, I I I}$ absorption edges was measured from a single crystal $\mathrm{Fe}$ film in the transverse configuration. The sample is a $35 \AA$ thick single crystal of $\mathrm{Fe}(110)$ ( 17 atomic layers ) deposited by molecular beam epitaxy on a (110) gallium arsenide substrate [6]. The effect of resonant excharge scattering manifests itself as changes in the specular reflectivity upon reversal of the direction of the nagnetic field. To illustrate the effect better, an asymmetry ratio $\mathbf{R}$ defined as $\mathbf{R}=\frac{\mathrm{I}^{+}-\mathrm{I}^{-}}{\mathrm{I}^{+}+\mathrm{I}^{-}}$was derived as a function of photon energy, where $\mathrm{I}^{+}$and $\mathrm{I}^{-}$ars the measured specular reflectivity with the applied magnetic field parallel and antiparellel to the cross product of the polarization vectors of the scattered and the incident beams.

Figure 1 shows the asymmetry ratios as well as $\mathrm{I}^{+}$and $\mathrm{I}^{-}$taken at a scattering angle of $35^{\circ}$. An asymmetry ratio as large as $13 \%$ was observed. The effect should be even larger at larger scattering angles because the ratio of the angular dependent 


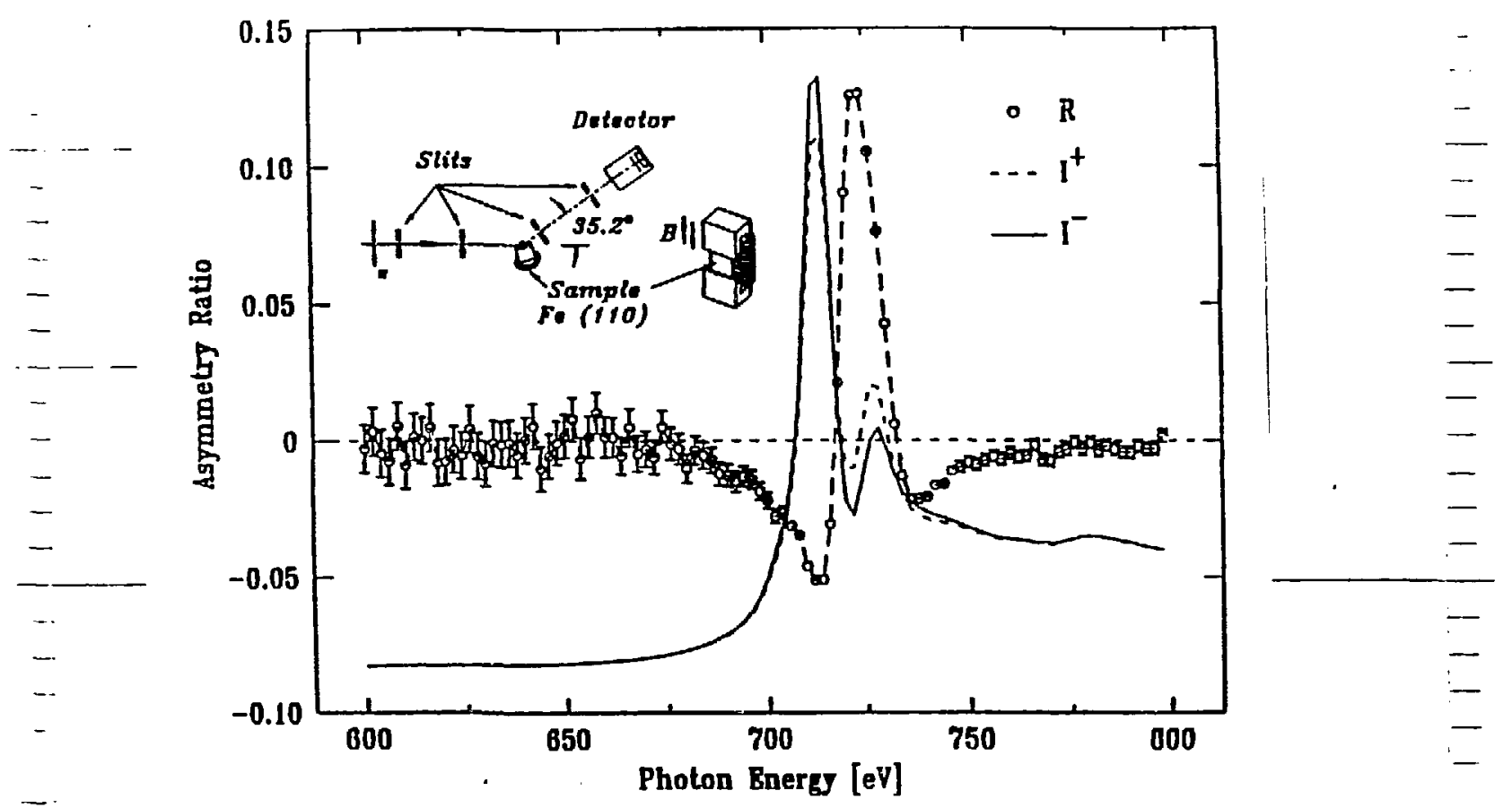

Fig. 1. Specular reflectivity of p-polarized light, $\mathrm{I}^{+}$and $\mathrm{I}^{-}$, and asymmetry ratios $\mathrm{R}$ measured from a $35 \AA \mathrm{Fe}(110)$ crystal.
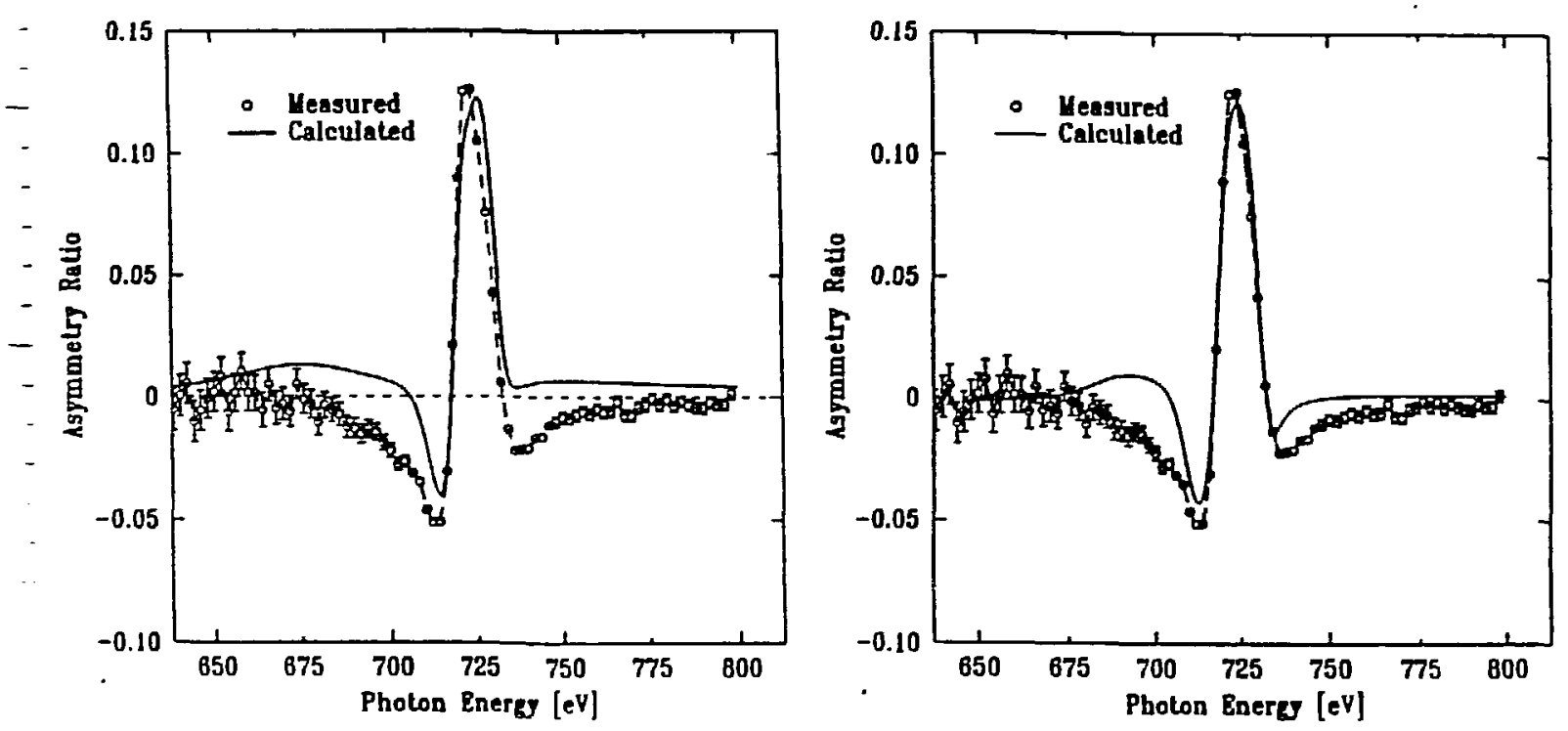

Fig. 2(a) Comparison between the measured and the calculated asymmetry ratios; 2(b) The same as 2(a) except a $5 \AA$ thick oxide layer is included in the calculation. 
parts, i.e. the ratio between the cross product and the dot product of the polarization vectors, is proportioal to $\tan (2 \theta)$.

The data agrees very well with an atomic-like model calculation based on the resonant exchange scattering process [7]. In this model, the $3 \mathrm{~d}$ states of $\mathrm{Fe}$ are treated as atomic levels with an exchange splitting $\Delta$. The resonant scattering amplitude then becomes

$$
f_{E 1}^{(r e s)}=F\left[\left(\vec{e}_{f} \cdot \vec{e}_{o}\right) n_{h}+i\left(\vec{e}_{f} \times \vec{e}_{o}\right) \cdot \vec{z}_{j} \frac{\mathbf{P}}{4}\right]
$$

with

$$
\mathbf{P}=\left[\mathbf{n}_{e}-\left(\frac{\Delta}{\Gamma(\mathbf{x}(\alpha, \eta)-i)}\right) \cdot \mathbf{n}_{\mathrm{h}}\right],
$$

where $\mathrm{F}$ includes the radial part of the transicion probability and the resonant denominator, $\frac{1}{x(\alpha, \eta)-i}, n_{e}$ is the net number of spin up electrons, $n_{h}$ is the total number of holes in the $3 d$ band, $\Gamma$ is the total width of the excited state, and $\Delta$ is the exchange splitting. In the resonant denominator $\mathbf{x}$ is the deviation from the absorption edge energy in units of haif widths, and is defined as

$$
\mathbf{x}(\alpha, \eta)=\frac{\left[\varepsilon-\left(E_{\eta}-E_{\alpha}\right)\right]}{\Gamma / 2},
$$

where $\varepsilon$ is the photon energy, and $\mathrm{E}_{\alpha}, \mathrm{E}_{\eta}$ are the energies of the ground state and the excited state respectively.

From equation (3), it is clear that the linear magnetic term is a function of both the spin polarization of the partially filled states and the exchange splitring of the unoccupied states. Given these functions, the specular reflecrivity and asymmerry ratios 
can then be calculated using the scattering amplitude in equation (2). Figure 2 (a) is a comparison of the calculated and the measured asymmetry ratios using the following parameters: $\mathbf{n}_{\mathrm{e}}=1, \mathbf{n}_{\mathrm{h}}=4, \Delta=1.5 \mathrm{eV}, \Gamma=5.0 \mathrm{eV}$. Better agreement between the calculated and the measured asymmetry ratios is obtained by including a $5 \AA$ thick surface oxide layer as shown in Figure 2 (b).

\section{Case II: Fe/Gd multilayer}

Asymmetry ratios were also measured for a $(32.4 \AA \mathrm{Fe} / 32.7 \AA)_{22}$ multilayer near both the Fe $\mathrm{L}_{\mathrm{II}, \mathrm{III}}$ edges and the $\mathrm{Gd} \mathrm{M}_{\mathrm{IV}, \mathrm{V}}$ edges as a function of photon energy and scattering angle [8]. Figure 3 shows the asymmetry ratios measured at $690 \mathrm{eV}$, about $17 \mathrm{eV}$ below the $\mathrm{L}_{\mathrm{III}}$ absorption threshold, as a function of wave vector transfer $\mathrm{Q}, 4 \pi \frac{\sin (\theta)}{\lambda}$, as well as the first order Bragg peak corresponding to the periodicity of the multilayer.

Since the wavelength of the photon is still much longer than the dimensions of the atomic states being excited, the standard Fresnel-Maxwell formulae used in the optical range are still applicable. Recently, several algorithms which allow arbitrary magnetization directions in the component layers have been developed to calculate the magneto-optical properties of these multilayer structures. For example, see Zak et al. [9] and references therein. In the following, a simpler algorithm specific to the transverse configuration used in the experiment is used to calculate the reflectivity of the $\mathrm{Fe} / \mathrm{Gd}$ multilayer.

Figure 4 (a) is a comparison of the calculated and the measured asymmetry ratios around the first Bragg peak at $690 \mathrm{eV}$, while figure 4 (b) shows the calculated asymmetry ratios at the same energy for an exiended range of $Q$. The agreement between the calculated and the measured asymmetry ratios in figure 4 (a) is not as good as in the case of the thin Fe film. However, the calculation does seem to reproduce the main features in the data: a major peak near the Bragg angle and a series of 


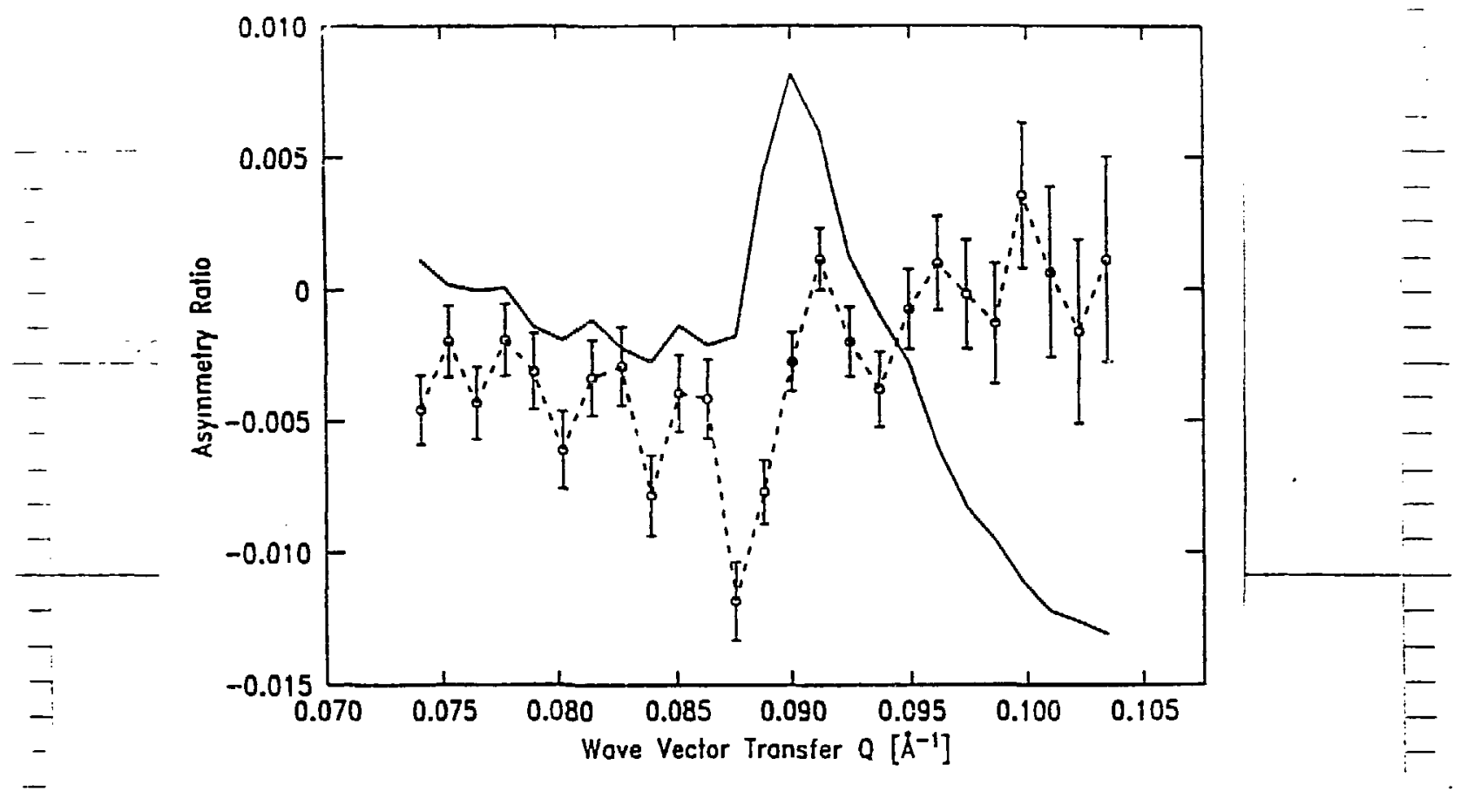

Fig. 3. Bragg scattering and asymmetry ratios from a ( $32.4 \AA \mathrm{Fe} / 32.7 \AA)_{22} \mathrm{mul}-$

- $\quad$ tilayer at $690 \mathrm{eV}$ photon energy.
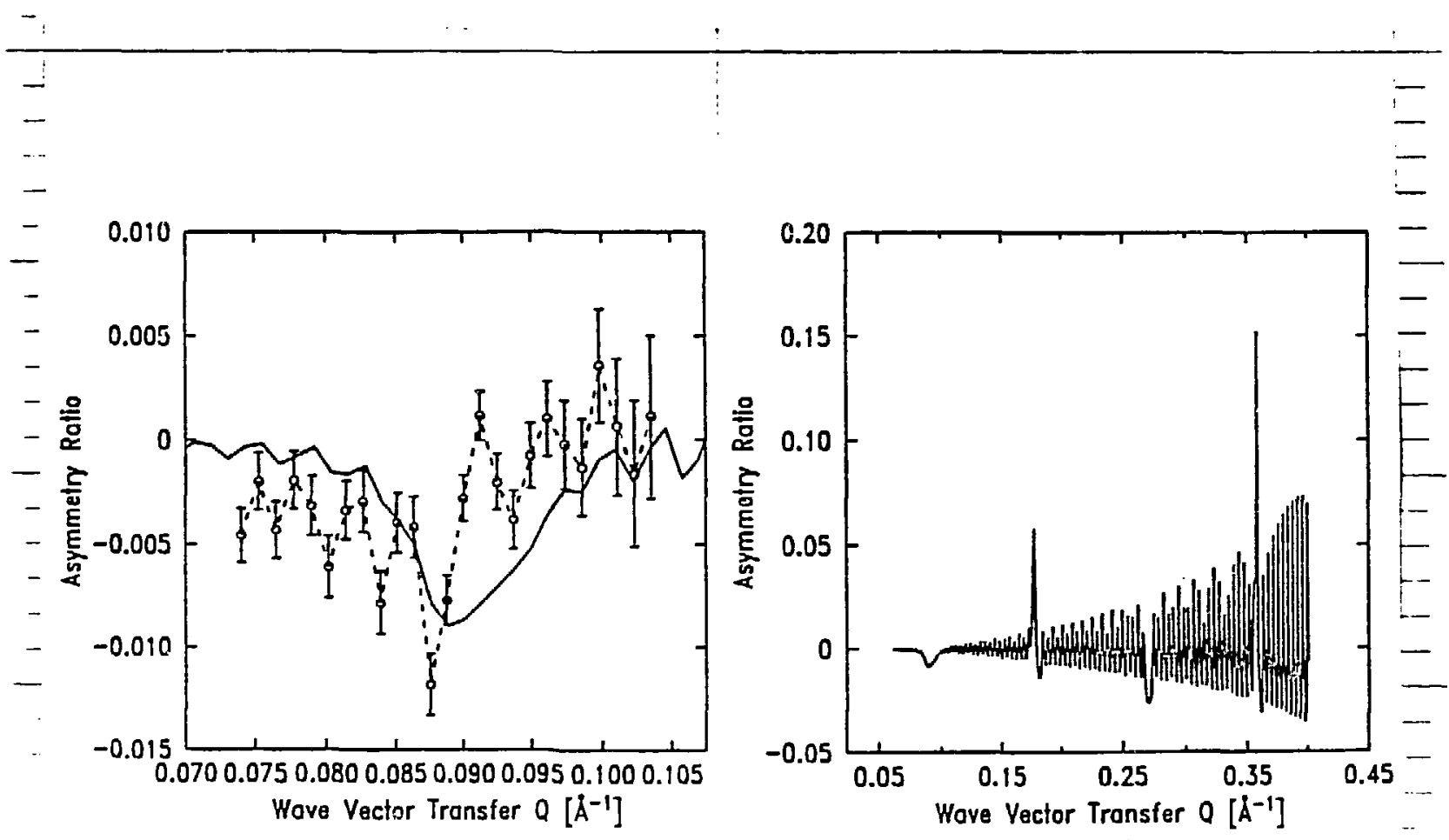

Fig. 4(a) Comparison between the measured and the calculated asymmetry ratios; 4(b) Calculated asymmetry ratios for an extended range of wave vector transfer $Q$. 
high frequency oscillations. The high frequency oscillations in the asymmetry ratios are due to the finite number of layers in the sample. The spacing between these oscillations agrees very well with sampling depth of 22 layers. This demonstrates that the penetration depth of the photon can be extended to over one thousand angstroms by tuning the photon energy to slightly below the absorption edge, since the effects of resonant magnetic scattering can be still quite large before the absorption edge. In fact, these oscillations can serve as a measure of the penetration depth of the photons. From figure 4 (b), it can be further stated that in addition to the angular dependence of the resonant scattering, as observed in the Fe film, further enhancement of the effect was observed at the Bragg angles.

\section{Conclusions}

(1)From the analysis of resonant magnetic scattering, the criteria for large enhancement of the magnetic scattering cross section near an absorption edge are the following: the absorption edge involves some strong mulipole transitions, large spinorbit coupling in the initial state to avoid partial cancellation of the effect, strong spin polarization of the unoccupied states, and large exchange splitting. In the soft $x$-ray region, large effect can be expected from $3 d$ transition ferromagnets and 4 f rare-earth magnetic materials.

(2)The results from the thin Fe film demonstrate that important spectroscopic information about the electronic structure of magnetic systems can be extracted from magnetic scattering measurements. Further, the information obtained from resonant magnetic scattering and related techniques, Faraday effect and magnetic circular dichroism, can be considered as complementary to that from spin-polarized photoemission.

(3)The large resonant enhancement combined with the high photon flux available from synchrotron radiation sources makes this technique especially attractive for the 
study of surface, interface, and thin film magnetism, and for the characterization of dilute magnetic samples.

(4)For multilayers consisting of $3 d$ and/or $4 f$ elements, large enhancement of the magnetic scattering cross section can be expected. Moreover, in cases where the wavelength of soft $\mathrm{x}$-ray photon used in the excitation is comparable to the periodicity of the multilayer, the ordering and coupling of these multilayers can also be studied. Field dependent and temperature dependent studies of these systems should provide valuable information on the understanding of these interesting systems.

This work was carried out in part under the auspices of DOE contract No. DEAC02-76CH00016.

\section{References}

1. D. Gibbs, D.R. Harshman, E.D. Isaacs, D.B. McWhan, D. Mills, and C. Vettier, Phys. Rev. Lett. 61, 1241 (1988).

2. E.D. Isaacs, D.B. McWhan, C. Peters, G.E. Ice, D.P. Siddons, J.B. Hastings, C. Vettier, and O. Vogt, Phys. Rev. Lett 62, 1671 (1989).

3. J.P. Hannon, G.T. Trammell, M. Blume, and D. Gibbs, Phys. Rev. Lett. 61, 1245 (1988), See also errata Phys. Rev. Lett. 62, 2644 (1989).

4. J.L. Erskine, E.A. Stem, Phys. Rev. B8, 1239 (1973); Phys. Rev. B12, 5016 (1975).

5. C.-C. Kao, D.P. Siddons, ( unpublished ).

6. Sample kindly provided by G.A. Prinz of NRL.

7. C.-C. Kao, J.B. Hastings, E.D. Johnson, D.P. Siddons, G.C. Smith, G.A. Prinz, Phys. Rev. Lett. 65, 373 (1990).

8. C.-C. Kao, E.D. Johnson, J.B. Hastings, C. Vettier, ( to be published ).

9. J. Zak, E.R. Moog, C. Liu, S.D. Bader, Phys. Rev. B43, 6423 (19G1). 


\section{DISCLAIMER}

This report was prepared as an account of work sponsored by an agency of the United States Government. Neither the United States Government nor any agency thereof, nor any of their employees, makes any warranty, express or implied, or assurizes any legal liability or responsibility for the accuracy, completeness, or usefulness of any information, apparatus, product, or process disclosed, or represents that its use would not infringe privately owned rights. Reference herein to any specific commercial product, process, or service by trade name, trademark, manufacturer, or otherwise does not necessarily constitute or imply its endorsement, recommendation, or favoring by the United States Government or any agency thereof. The views and opinions of authors expressed herein do not necessarily state or reflect those of the United States Government or any agency thereof. 